\title{
Neurosurgical Focus
}

\section{Volume 6, Issue 2}

\section{February 1999}

\section{Medical and Surgical Control of Epilepsy}

\section{Introduction by Topic Editor: Dennis Spencer, M.D., Harvey and Kate Cushing Professor and Chairman, Department of Neurosurgery, Yale University School of Medicine}

The tendency for the human brain to suffer seizures, either as focal excitation, recruiting additional neurons and glia, or a diffuse uncontrolled discharge, has been historically documented as one of the oldest and most dramatic afflictions of man. Epilepsy remains the most common primary disorder of the central nervous system. As in Horsely's first neurosurgical case for tumors focal seizures have value as a localizing phenomenon, but for the majority of this century only a few centers have devoted surgical attention to the treatment of epilepsy as a syndrome.

Epilepsy afflicts $1 \%$ of the population and is a major health problem worldwide. Despite exhaustive pharmacological research, drugs are unable to control the symptoms of all of those afflicted, leaving a group of approximately 250,000 patients with symptomatic epilepsy who may be benefited by a variety of surgical approaches.

Given the heterogeneous nature of the epilepsy population and the lack of a single pathogenic mechanism, the slow growth in this field is understandable. In fact, it is increasingly clear that various genetic aberrations may affect many disparate neural pathways to converge in ways that lower the brain threshold for excitability.

During the first three quarters of this century, the search for focal, resectable, brain regions responsible for seizures was guided by both noninvasive and invasive electrophysiology. This labor intensive search coupled with the reluctance of medical physicians to recommend such apparently radical treatment for their patients, dictated slow progress in surgical therapy.

During the decade of the 1980's, however, computers revolutionized imaging and electrophysiological techniques, and there was incredible growth in the number of epilepsy surgery centers. During this era magnetic resonance (MR) imaging, coupled with 24 hour audiovisual EEG, unveiled important 
correlations of lesions with epileptogenicity. It became clear that the lobar classification of the symptomatic epilepsies, (i.e. temporal lobe epilepsy, and frontal lobe epilepsy), should be reconsidered, and perhaps replaced by a classification based on pathogenetic substrates.

We began to assume that many surgically remediable epilepsy disorders had a pathological origin that was defined by MR images or discovered in the resected microscopic histology. The epileptogenic substrate, therefore, would appear to consist of tissue pathology interacting with a particular cortical region's behavioral expression of the resultant neural excitability. We now know that the majority of the substrates causing chronic epilepsy are congenital in origin and consist of vascular lesions such as cavernous angiomas and artereovenous malformations, developmental abnormalities, such as cortical displasia, hamartomas, and heterotopias and the tumors such as gangliogliomas, and dysembryoplastic epithelial tumors. Even the sclerotic lesions with neuronal cell loss and gliosis (mesial temporal sclerosis) have evidence of genetic predisposition. Knowledge of genetic eleptogenic origins and resulting lesions is so new it has devalued a previous body of knowledge upon which the epileptologist and surgeon could turn to in order to best match patients to particular procedures or predict the outcome of epilepsy. Magnetic resonance imaging has allowed identification of these epileptogenic substrates which has greatly broadened our surgical experience, but has not provided new srategies for treatment.

The major theme of this issue of Neurosurgical Focus reflects that this vacuum in our literature is beginning to be filled. The decade of the 1990's has not seen growth of many new procedures for epilepsy, but has been a period of consolidation where most centers are searching to optimize both standard drug and surgical treatment. We have therefore selected papers dealing with surgical outcome and the empirical data that might best predict success in surgical control. Readers will note that topics range from the evaluation of a population at risk for seizures (Paper one), the impact of an acute postoperative seizure on the prediction of seizure control (Paper two) and then to an outcome study of lesional surgery in children that is retrospective, but takes appropriate advantage of multivariate analysis to make some simple important points (Paper three). Despite the growth and incalculable assistance of MR imaging, the logic tree that guides the surgeon to a particular therapy often remains obfuscated with many variables. Paper four addresses the unique concept of using computerized neural networks that learn through feedback to pick critical predictive data. This is a controversial field, and Assistant Editor David Roberts provides a comment for perspective. Finally we have included a paper on the new technology of functional MR imaging that will hopefully enhance our ability to determine how developmental injuries causing epilepsy interact with normal function, how we can predict where their function resides and eventually coregister maps of pathology, electrophysiology, and biochemical alterations and cognitive function. Our goal, as pointed out in Paper three, is to acquire the skills for complete resection of pathological tissue without harm to our patients.

I would like to thank my-coeditors, David Roberts and Richard Morwitz for their excellent assistance. 